\title{
DYNAMICAL FRICTION AND ORBIT CIRCULARIZATION
}

\author{
Stefano Casertano \\ Institute for Advanced Study, Princeton \\ E. Sterl Phinney and Jens V. Villumsen \\ Caltech, Pasadena
}

We study the change in shape of the orbit of a satellite sinking (because of dynamical friction) towards the center of a larger galaxy. The galaxy is assumed spherically symmetric with distribution function either isotropic or predominantly radial. The satellite is a softened point mass.

The orbit evolution is studied analytically using the Chandrasekhar (1943) approximation for the dynamical friction drag and the epicyclic approximation for the orbit of the satellite, and numerically, by direct integration of an N-body system.

Fully self-consistent spherical models have been used for the galaxy. All the models have a King (1966) density profile with concentration parameter 1.255. Anisotropic models are constructed with the prescriptions of Merritt (1985). For these models the ratio of radial to tangential velocity dispersions follows the law $\sigma_{r}^{2} / \sigma_{t}^{2}=1+r^{2} / r_{a}^{2}$; they are isotropic in the center and strongly radially anisotropic for $r \gg r_{a}$.

The analytical treatment gives an explicit form for the function $\Upsilon(r) \equiv$ $d \ln (\epsilon) / d \ln (r)$ involving integrals over the distribution function. Although the explicit form of $\Upsilon(r)$ is rather complicated, numerical evaluation is straightforward. Some general properties of $\Upsilon(r)$ are:

- $\Upsilon(r) \geq 0$ (implying circularization) for any realistic isotropic system.

- For many realistic isotropic systems, $\Upsilon(r) \sim 0.5$ for $r \gg r_{c}$.

- $\Upsilon(r) \rightarrow 0$ as $r \rightarrow r_{c}$.

- Increasing the radial anisotropy makes $\Upsilon(r)$ smaller.

The dashed lines in Fig. 1 give the theoretical $\epsilon(r)$ for an isotropic system (a) and for a marginally unstable system with $r_{a}=2 r_{c}$ (b). For even more anisotropic systems $\left(r_{a} \leq 1.5 r_{c}\right), \Upsilon(r)$ may be negative, corresponding to orbits becoming less circular as they shrink. However, such systems are unstable to bar formation (Barnes 1985).

The validity of the analytic treatment has been tested by a number of N-body simulations with the large galaxy made up of 5000-20000 points, and the satellite being treated as a point mass. The self-gravity of the galaxy is computed by spherical harmonics expansion, whereas the galaxy-satellite interaction is treated by direct summation over the particles. In agreement with the analytical predictions, the eccentricity decreases significantly in the isotropic system, but not for the anisotropic case (see Fig. 1). 

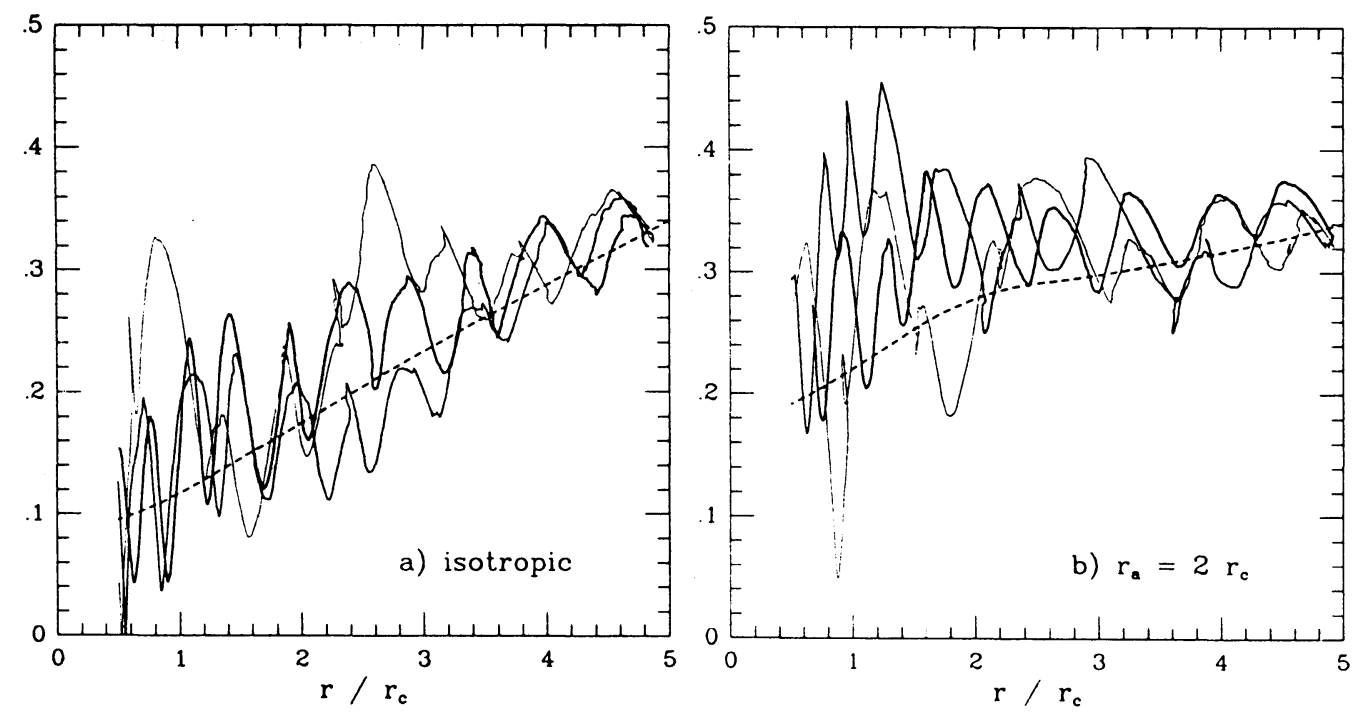

Figure 1. Predicted (dashed) and numerical (solid) variations of eccentricity with radius. Panel a) refers to an isotropic King model, panel b) to an anisotropic model with the same density distribution. The $\mathrm{N}$-body simulations have $\mathrm{N}=\mathbf{2 0 0 0 0}$ (thick solid), 10000 (medium) and 5000 (thin).

The eccentricity calculated from the numerical experiments is however very noisy, and the agreement is only semi-quantitative. From a detailed comparison of predicted and calculated eccentricity, we find:

- In the isotropic case, $\epsilon$ decreases appreciably. The corresponding value of $\Upsilon$ is not well determined, but is within $\mathbf{5 0 \%}$ of the analytical prediction.

- There is no measurable decrease in eccentricity in the anisotropic case $\left(r_{a}=\right.$ $2 r_{c}$ ), consistent with our predictions.

\section{REFERENCES}

Barnes, J. 1985, unpublished.

Chandrasekhar, S. 1943, Ap. J. 97, 255.

King, I. R. 1966, A. J. 71, 64.

Merritt, D. 1985, A. J. 90, 1027. 